\title{
3D Pattern Synthesis of Time-Modulated Conformal Arrays with a Multiobjective Optimization Approach
}

\author{
Wentao Li, ${ }^{1}$ Yongqiang Hei, ${ }^{2}$ Jing Yang, ${ }^{1}$ and Xiaowei Shi ${ }^{1}$ \\ ${ }^{1}$ School of Electronic Engineering, Xidian University, Xian, Shaanxi 710071, China \\ ${ }^{2}$ State Key Laboratory of Integrated Services Networks, Xidian University, Xian, Shaanxi 710071, China \\ Correspondence should be addressed to Wentao Li; wtli@mail.xidian.edu.cn
}

Received 28 November 2013; Revised 24 April 2014; Accepted 28 April 2014; Published 2 June 2014

Academic Editor: Atsushi Mase

Copyright (c) 2014 Wentao Li et al. This is an open access article distributed under the Creative Commons Attribution License, which permits unrestricted use, distribution, and reproduction in any medium, provided the original work is properly cited.

\begin{abstract}
This paper addresses the synthesis of the three-dimensional (3D) radiation patterns of the time-modulated conformal arrays. Due to the nature of periodic time modulation, harmonic radiation patterns are generated at the multiples of the modulation frequency in time-modulated arrays. Thus, the optimization goal of the time-modulated conformal array includes the optimization of the sidelobe level at the operating frequency and the sideband levels (SBLs) at the harmonic frequency, and the design can be regarded as a multiobjective problem. The multiobjective particle swarm optimization (MOPSO) is applied to optimize the switchon instants and pulse durations of the time-modulated conformal array. To significantly reduce the optimization variables, the modified Bernstein polynomial is employed in the synthesis process. Furthermore, dual polarized patch antenna is designed as radiator to achieve low cross-polarization level during the beam scanning. A $12 \times 13$ (156)-element conical conformal microstrip array is simulated to demonstrate the proposed synthesis mechanism, and good results reveal the promising ability of the proposed algorithm in solving the synthesis of the time-modulated conformal arrays problem.
\end{abstract}

\section{Introduction}

Conformal phased arrays will prove their potential applications in a variety of fields, such as airborne, missile, and unmanned aerial vehicles, due to their excellent aerodynamic performances [1-3]. However, due to the influences of curved carriers, the design of conformal arrays also faces many challenges. Unlike planar and linear arrays, the radiating elements of conformal arrays generally orient in different directions on curved surfaces, posing unique challenges in the synthesis of antenna arrays. In particular, the crosspolarization level of the conformal phased array is more serious compared with the planar or linear arrays.

With the development of high-speed computers, the synthesis of conformal arrays has made a series of progresses. Bucci et al. proposed a conformal array synthesis procedure which incorporates near-field constraints [4]. In 2005 , Boeringer and Werner presented an approach to curved phased array synthesis using the particle swarm optimization with a modified Bernstein polynomial to obtain the excitation amplitudes [5]. However, during the design process, many practical factors were not considered. In 2007, Vaskelainen used a constrained least-squares method to synthesize conformal arrays and the least mean square (LMS) error of the constrained least-squares optimization problem has been studied [6]. In 2010, Fuchs proposed a procedure for the synthesis of shaped beams, which reduced the synthesis problem to a linear programming one. A cylindrical array of $8 \times 8$ elements was utilized to illustrate the effectiveness of the method, but the optimality of the solution could not be guaranteed [7]. Similarly, Tsui and Chan used iterative second-order cone programming method for the power or shaped beam pattern synthesis of narrowband conformal arrays [8].

However, for the synthesis of patterns with low/ultralow sidelobes of the conventional conformal arrays, the obtained dynamic range ratios of amplitude excitations could be quite high, which results in stringent requirements on various error tolerances for practical implementation. With the rapid development of engineering technology, the 
previously proposed time-modulated antenna array (TMAA) causes the researchers' attention again [9]. By introducing a fourth dimension-time-into conventional arrays, the feed network of the antenna array can be simplified greatly. Motivated by this, introducing the "time-modulated" technology into the conformal phased array will provide a new design approach.

Compared with the conventional array, in time-modulated array, each antenna element is connected with a highspeed RF switch which works periodically. Due to the periodic modulation of switches, the inherent property of time-modulated antenna arrays is the presence of sidebands or harmonic signals at the multiples of the time modulation frequency. The sideband signals result in a reduction of the array gain. Aiming at improving the gain of TMAAs and suppressing the sideband levels (SBL), various stochastic optimization algorithms have been used, such as the particle swarm optimization (PSO), genetic algorithm (GA), simulated annealing, differential evolution algorithm, and artificial bee colony algorithm [10-13]. Recently, to improve the gain of TMAAs, a novel approach is proposed by using single-pole-double-throw switches [14], and the concept of a time-modulated reflector array has been analyzed in [15]. In [16], the pulse shaping has been employed as an additional degree of freedom to yield a nonnegligible effect on reduction of the sideband losses. Furthermore, the total power radiated in harmonics for asymmetric pulse distributions has been studied in [17]. However, sideband signals can also be utilized. At different sideband frequencies, the time-modulated antenna array can produce multiple beams simultaneously and point at different angular directions $[18,19]$.

In this paper, the synthesis of the three-dimensional (3D) radiation patterns in full space of the time-modulated conformal arrays is investigated. Due to the multiobjective nature of the optimization problem, considering both the SLL at the operating frequency and the sideband levels (SBLs), simultaneously, the multiobjective particle swarm optimization (MOPSO) is employed. To reduce the optimization variables and accelerate the speed of convergence, the modified Bernstein polynomial is extended to $3 \mathrm{D}$ case in synthesizing conformal arrays. Furthermore, in order to control the polarization of the array during scanning, a dual fed stacked microstrip antenna is designed to build the conical conformal array. Finally, a $12 \times 13$ (156)-element conical conformal phased array has been successfully simulated.

The remainder of the paper is organized as follows. Section 2 briefly explains the formulation of the problem. Section 3 presents the detailed architecture of the proposed algorithm. Numerical results are discussed in Section 4, while Section 5 concludes this paper.

\section{Problem Formulation}

Consider an array of $M \times N$ elements located over a conical surface, as shown in Figure 1, where $M$ and $N$ represent the number of elements in the $z$-direction and $\varphi$-direction, respectively. The radii of the cone at the top and bottom are denoted as $r_{1}=2.29 \lambda$ and $r_{2}=3.85 \lambda$, respectively. The height of the cone is $h=5.48 \lambda$ and the half cone angle is $\theta_{c}=16^{\circ}$. The elements are arranged according to the following rule: the space between consecutive horizontal rows is $d_{z}=0.5 \lambda$ in the $z$-direction; that is, the elements in the same column are along the same generatrix; the distance between the adjacent elements on the bottom layer is $d_{l}=0.67 \lambda$.

The far-field radiation pattern produced by these $M \times N$ elements placed on the conical surface can be expressed as

$$
\begin{aligned}
\mathrm{FF}(\theta, \varphi)= & \sum_{m=1}^{M} \sum_{N=1}^{N} I_{m n} f_{m n}(\theta, \varphi) \\
& \times \exp \left[j k r _ { n } \left[\sin \theta_{c} \sin \theta \cos \left(\varphi-\varphi_{m n}\right)\right.\right. \\
& \left.\left.\quad-\cos \theta \cos \theta_{c}\right]+j \psi_{m n}\right],
\end{aligned}
$$

where $I_{m n}$ is the element excitation current amplitude, $\psi_{m n}$ is the excitation current phase, $f_{m n}(\theta, \varphi)$ is the individual element pattern, and $r_{n}$ is the radius of circle, on which the $m n$th element is placed. $k$ is the free-space wave number. The excitation current phase $\psi_{m n}$ can be calculated by

$$
\psi_{m n}=-k r_{n}\left[\sin \theta_{c} \sin \theta_{0} \cos \left(\varphi_{0}-\varphi_{m n}\right)-\cos \theta_{c} \cos \theta_{0}\right]
$$

where $\left(\theta_{0}, \varphi_{0}\right)$ is the desired steering angle.

In time-modulated arrays, each element is controlled by a high speed RF switch which works periodically. In each modulation period $T_{p}$, the switch-on time interval is $\tau_{n}(0 \leq$ $\left.t \leq T_{p}\right)$ and the time-modulated frequency is $f_{p}=1 / T_{p}$.

Such switch can be represented by a unit step function $U_{m n}(t)$ depicted as

$$
U_{m n}(t)= \begin{cases}1, & 0 \leq t \leq \tau_{m n} \\ 0, & \text { otherwise. }\end{cases}
$$

The far-field radiation pattern of the conical conformal array in (1) can be rewritten as

$$
\begin{aligned}
\operatorname{FF}(\theta, \varphi, t)= & \sum_{m=1}^{M} \sum_{N=1}^{N} I_{m n} U_{m n}(t) f_{m n}(\theta, \varphi) \\
& \times \exp \left[j k r _ { n } \left[\sin \theta_{c} \sin \theta \cos \left(\varphi-\varphi_{m n}\right)\right.\right. \\
& \left.\left.-\cos \theta \cos \theta_{c}\right]+j \psi_{m n}\right] .
\end{aligned}
$$

Since $U_{m n}(t)$ is a continuous time periodic function, its Fourier series exists and (4) can be decomposed into a Fourier series with different frequency components as follows:

$$
\begin{aligned}
\mathrm{FF}(\theta, \varphi, t)= & \sum_{q=-\infty}^{\infty} e^{j 2 \pi\left(f_{0}+q f_{p}\right) t} \\
& \times \sum_{m=1}^{M} \sum_{N=1}^{N} I_{m n} a_{q m n} f_{m n}(\theta, \varphi) \\
& \times \exp \left[j k r _ { n } \left[\sin \theta_{c} \sin \theta \cos \left(\varphi-\varphi_{m n}\right)\right.\right. \\
& \left.\left.\quad-\cos \theta \cos \theta_{c}\right]+j \psi_{m n}\right],
\end{aligned}
$$




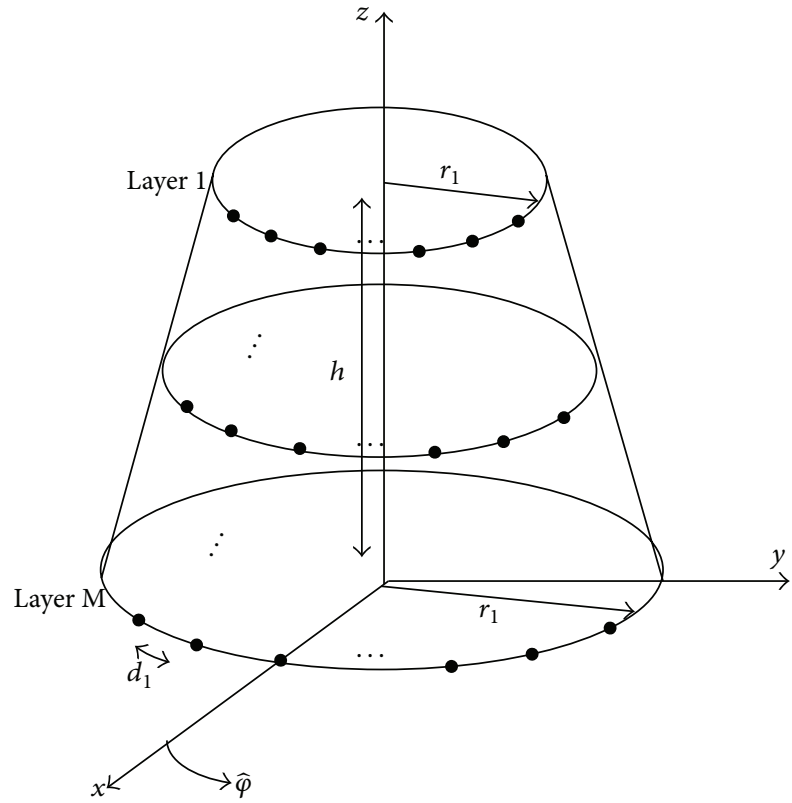

(a)

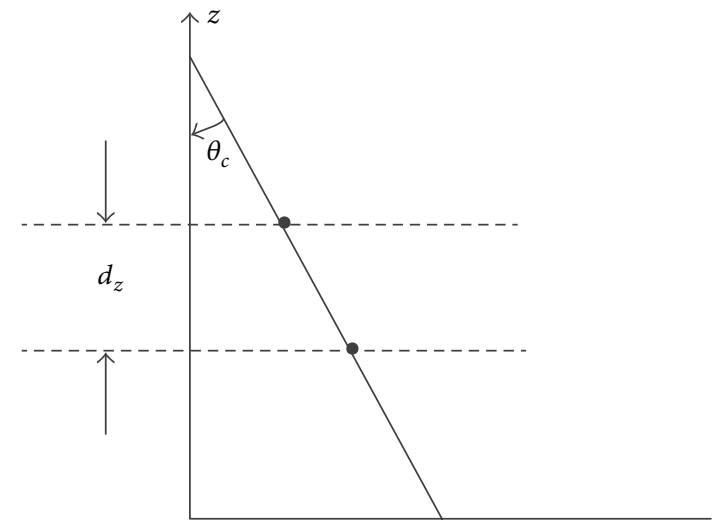

(b)

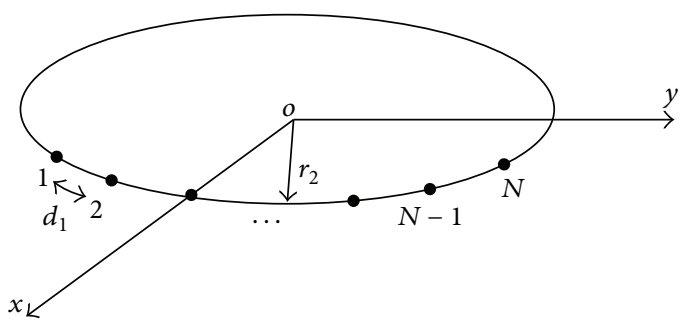

(c)

FIgURE 1: A graphical representation of the antenna element distribution conformal to the conical surface. (a) 3D structure. (b) Sectional View. (c) Cross sectional view for the bottom ring.

where $f_{0}$ is the operating frequency. The $q$ th-order $(q=$ $0, \pm 1, \pm 2, \ldots, \pm \infty$ ) Fourier component is

$$
\begin{aligned}
\left.\mathrm{FF}(\theta, \varphi, t)\right|_{q}= & e^{j 2 \pi\left(f_{0}+q f_{p}\right) t} \\
& \times \sum_{m=1}^{M} \sum_{N=1}^{N} I_{m n} a_{q m n} f_{m n}(\theta, \varphi) \\
& \times \exp \left[j k r _ { n } \left[\sin \theta_{c} \sin \theta \cos \left(\varphi-\varphi_{m n}\right)\right.\right. \\
& \left.\left.\quad-\cos \theta \cos \theta_{c}\right]+j \psi_{m n}\right],
\end{aligned}
$$

where the amplitude for the $q$ th harmonic $a_{q m n}$ is integrated as

$$
a_{q m n}=f_{p} \tau_{m n} \frac{\sin \left(q \pi f_{p} \tau_{m n}\right)}{q \pi f_{p} \tau_{m n}} e^{-j q \pi f_{p} \tau_{m n}} .
$$

Obviously, the expression of the far-field radiation pattern at the operating frequency is determined by the $q=0$ component as follows:

$$
\begin{aligned}
\left.\mathrm{FF}(\theta, \varphi, t)\right|_{q=0}= & e^{j 2 \pi f_{0} t} \\
& \times \sum_{m=1}^{M} \sum_{N=1}^{N} \frac{I_{m n} \tau_{m n}}{T_{p}} a_{q m n} f_{m n}(\theta, \varphi) \\
& \times \exp \left[j k r _ { n } \left[\sin \theta_{c} \sin \theta \cos \left(\varphi-\varphi_{m n}\right)\right.\right. \\
& \left.\left.\quad-\cos \theta \cos \theta_{c}\right]+j \psi_{m n}\right] .
\end{aligned}
$$

Since time-modulated array radiates at each harmonic frequency, it is necessary to suppress the sideband level for the purpose of reducing the energy loss and the interference. From (7), we can see that $a_{q m n}$ obeys the $\sin (x) / x$ distribution. Therefore, it is usually needed to suppress the maximum sideband level at the first sideband frequency $f_{0}+f_{p}$. However, recent study shows that minimizing the first sideband level may produce inaccurate results [20] and therefore at least a 


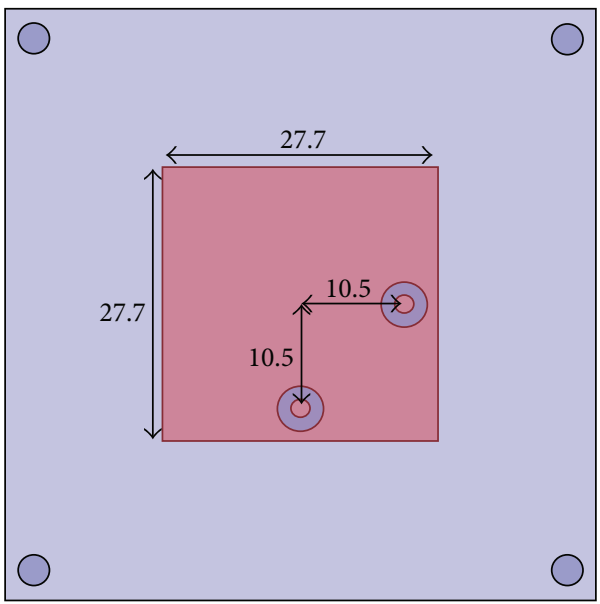

(a)

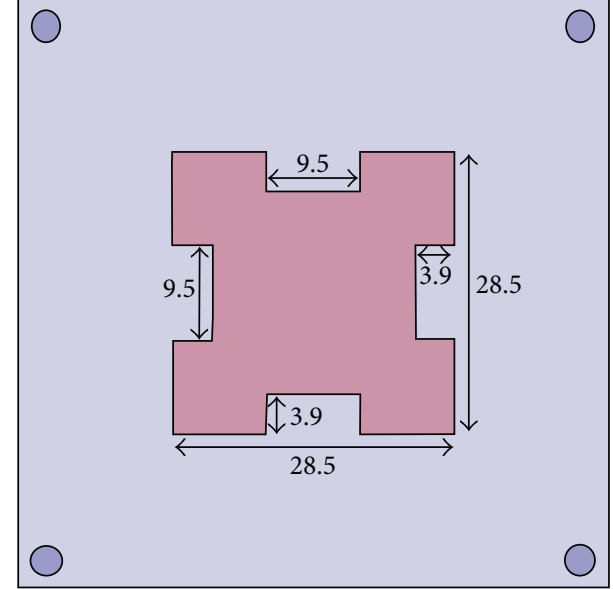

(b)

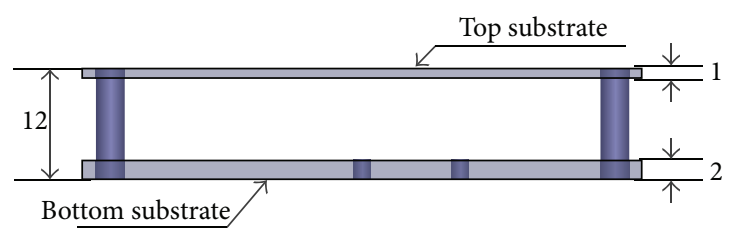

(c)

Figure 2: Configurations of the stacked microstrip antenna. (a) Top view of bottom layer. (b) Top view of top layer. (c) Side view (all units are in $\mathrm{mm}$ ).

few harmonics should be taken into account to ensure the sideband level suppression is conducted.

\section{MOPSO Algorithm}

3.1. Terminologies of Multiobjective Optimizations. Without loss of generality, the following minimization problem is to be considered:

$$
\begin{array}{ll}
\text { Minimize } & \vec{f}(\vec{x})=\left\{f_{1}(\vec{x}), f_{2}(\vec{x}), \ldots, f_{M}(\vec{x}),\right. \\
& m=1,2, \ldots, M\}, \\
\text { subject to constraints } & g_{i}(\vec{x}) \leq 0 ; \quad i=1,2, \ldots, p \\
& h_{i}(\vec{x})=0 ; \quad i=1,2, \ldots, q,
\end{array}
$$

where $\vec{x}=\left\{x_{i}, i=1,2, \ldots, D\right\}$ is a vector of decision variables. These constraints define the feasible region, and any vector in the feasible region is called a feasible solution.

In multiobjective optimal problems, the following two terminologies are often used.

Dominance Relations. The definitions of dominance relations between two vectors (or individuals of the population) are given in [21]. The weak dominance relation $(\preceq)$ between $\vec{x}_{1}$ and $\vec{x}_{2}$ is defined as:

$\vec{x}_{1}$ weak dominates $\vec{x}_{2} \quad \vec{x}_{1} \preceq \vec{x}_{2} \quad$ iff $\forall i: f_{i}\left(\vec{x}_{1}\right) \leq f_{i}\left(\vec{x}_{2}\right)$ while the dominance relation $(\prec)$ is defined as

$$
\begin{gathered}
\vec{x}_{1} \text { dominates } \vec{x}_{2} \quad \vec{x}_{1} \prec \vec{x}_{2} \\
\text { iff } \vec{x}_{1} \preceq \vec{x}_{2} \wedge \exists i: f_{i}\left(\vec{x}_{1}\right)<f_{i}\left(\vec{x}_{2}\right) .
\end{gathered}
$$

The dominance relationship can be extended to take into consideration constraint values besides objective values; the relation constraint-dominance $\left(<_{c}\right)$ is defined as: $\vec{x}_{1}$ constraint-dominates $\vec{x}_{2} \vec{x}_{1} \prec_{c} \vec{x}_{2}$, if any of the following conditions is true.

(1) $\vec{x}_{1}$ belongs to the feasible space and $\vec{x}_{2}$ does not.

(2) $\vec{x}_{1}$ and $\vec{x}_{2}$ are both infeasible and $\vec{x}_{1}$ dominates $\vec{x}_{2}$ in constraint function space.

(3) $\vec{x}_{1}$ and $\vec{x}_{2}$ are feasible and $\vec{x}_{1}$ dominates $\vec{x}_{2}$ in objective function space.

Pareto Optimal (Solution, or Front). The strongly and weakly nondominated solutions constitute the total Pareto front of a multiobjective optimization problem.

3.2. MOPSO Algorithm. Particle swarm optimization (PSO) is a stochastic population-based multipoint search optimization technique developed by Eberhart and Kennedy in 1995 [22], inspired by the social behavior of bird flocking or fish schooling. In PSO, each particle has a position vector $\vec{x}_{i}=\left(x_{i 1}, x_{i 2}, \ldots, x_{i D}\right)$ in the $D$-dimensional search space and moves through the problem space, with the moving velocity of each particle represented by a position vector 


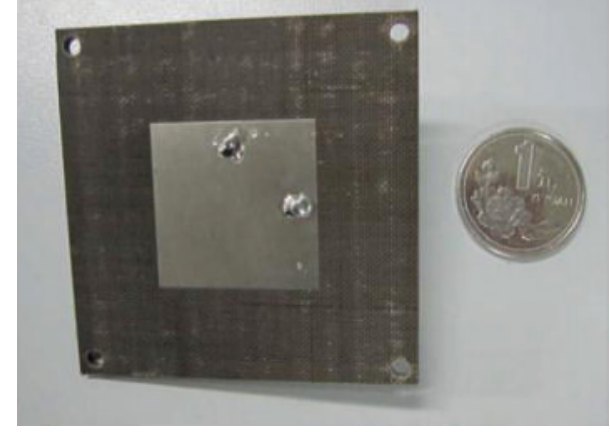

(a)

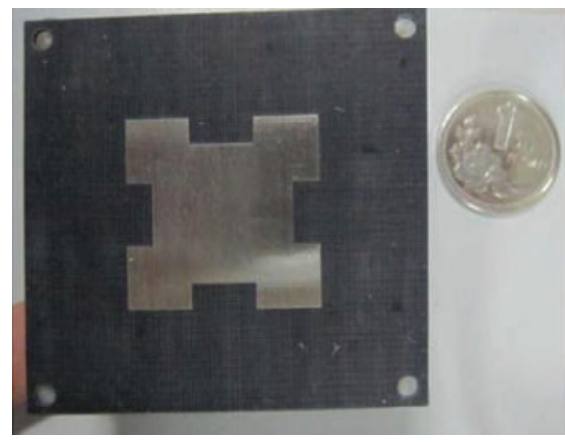

(b)

FIgURe 3: Photograph of the fabricated antenna. (a) Top layer. (b) Bottom layer.

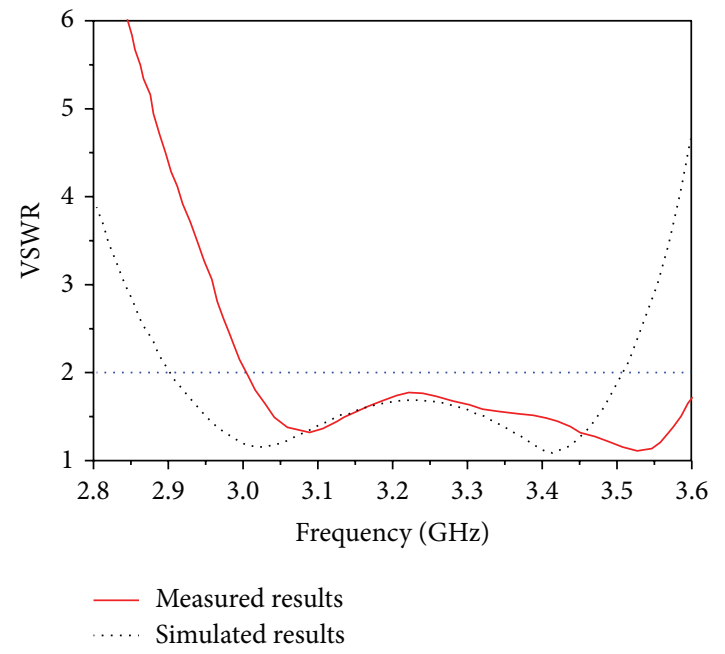

FIGURE 4: Simulated and measured VSWR for the antenna.

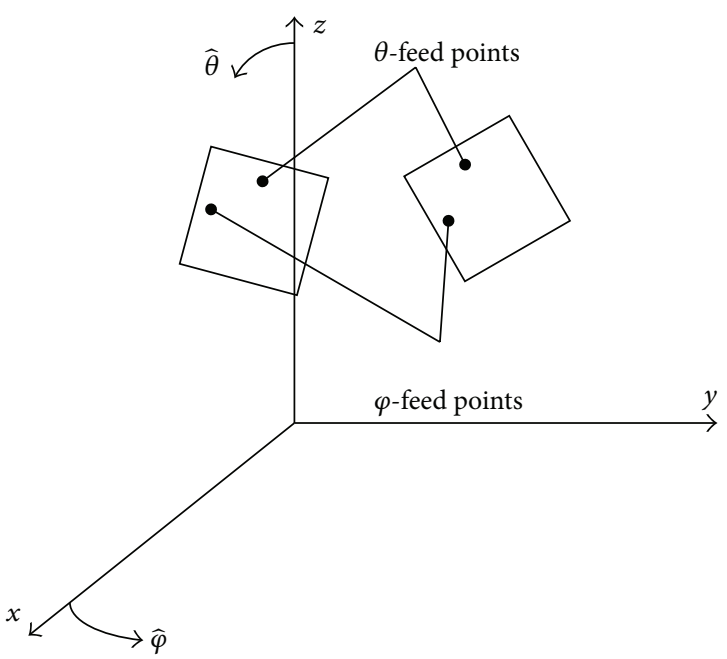

FIGURE 5: The schematic diagram of the antennas positioned on the conical surface with the $\theta$-feed points in the $\widehat{\theta}$-direction and the $\varphi$ feed points in the $\hat{\varphi}$-direction.

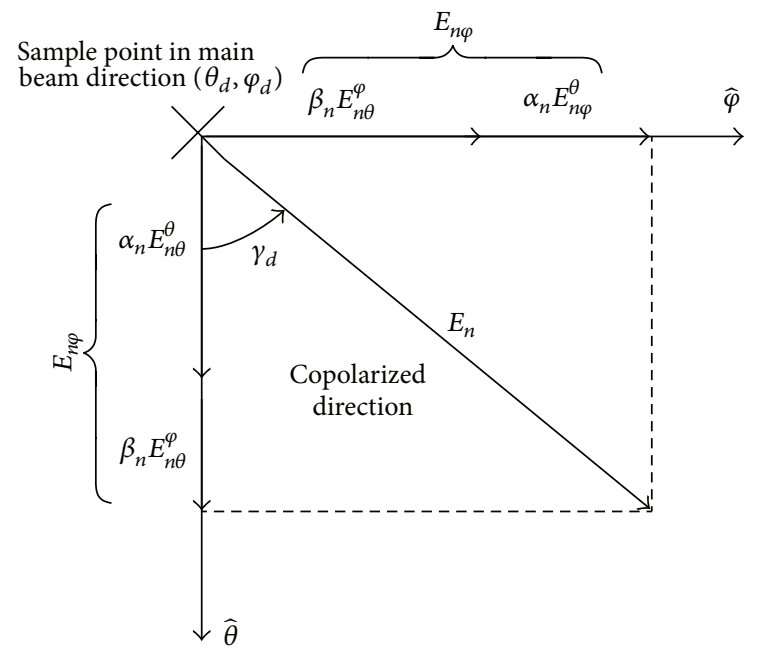

FIGURE 6: A graphical representation of the relations between the desired polarization vector in the main beam direction and the various components of the radiated field.

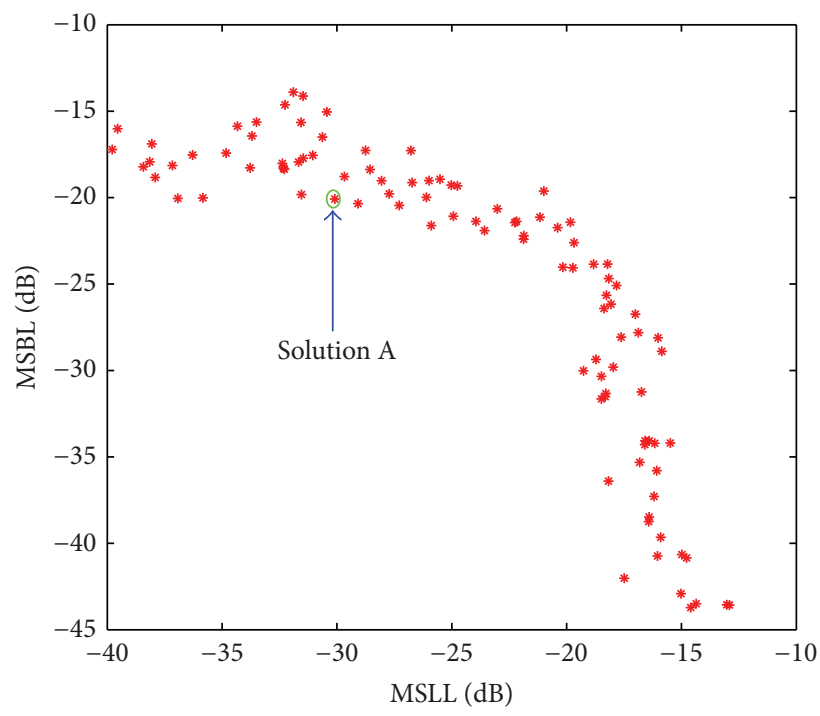

FIGURE 7: Pareto front obtained by the proposed MOPSO for scan direction of $\left(74^{\circ}, 0^{\circ}\right)$. 


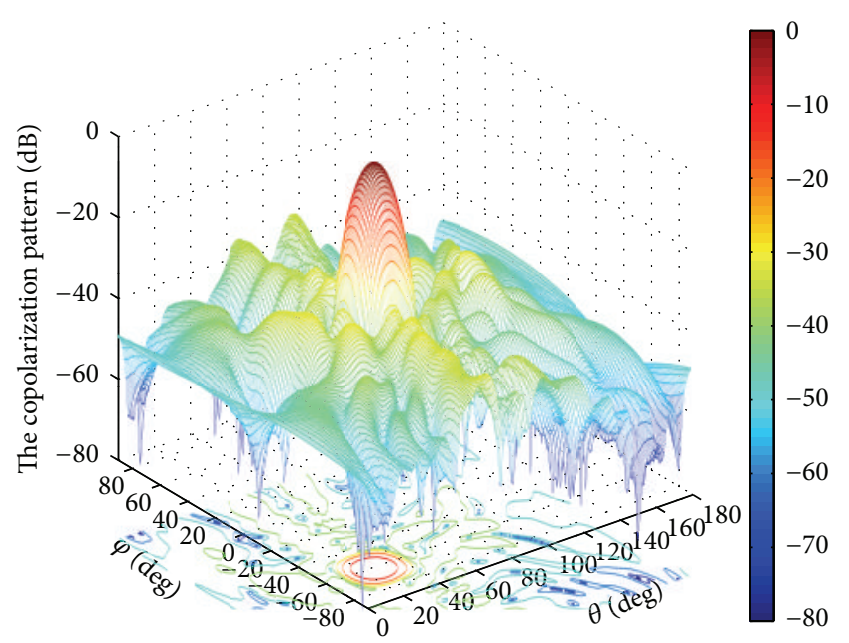

(a) Copolarization pattern

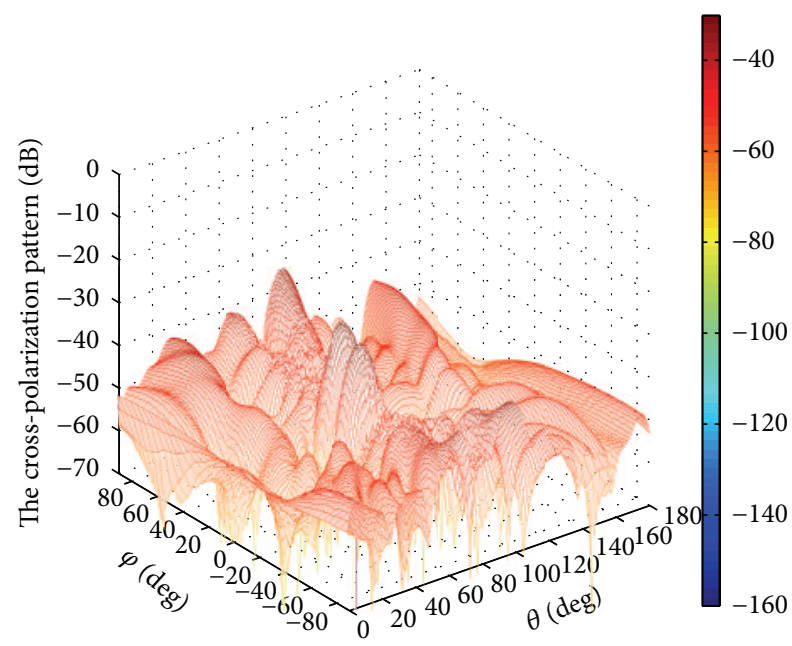

(b) Cross-polarization pattern

FIGURE 8: 3D radiation pattern of the conical conformal array for scan direction of $\left(74^{\circ}, 0^{\circ}\right)$ for solution A in Figure 7.

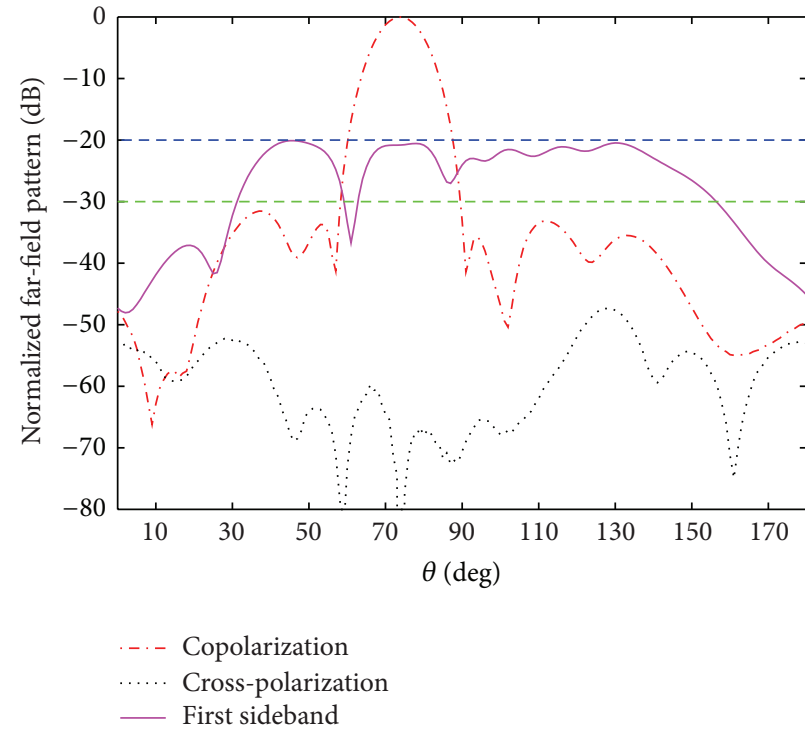

(a) $\varphi=0^{\circ}$ plane

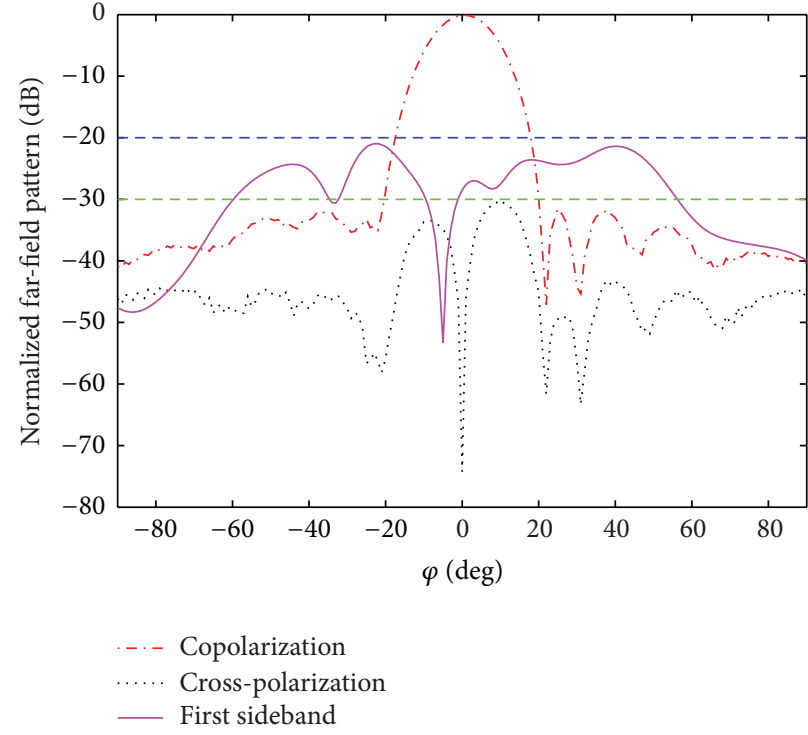

(b) $\theta=74^{\circ}$ plane

Figure 9: Sectional views in the elevation and azimuth planes for scan direction of $\left(74^{\circ}, 0^{\circ}\right)$ for solution A in Figure 7.

$\vec{v}_{i}=\left(v_{i 1}, v_{i 2}, \ldots, v_{i D}\right)$. Each particle keeps track of its own best position pbest $_{i}=\left(\right.$ best $_{i 1}$, best $_{i 2}, \ldots$, pbest $\left._{i D}\right)$ and the best position among all the particles obtained so far in the population $g$ best $=\left(\right.$ gbest $_{1}$, gbest $_{2}, \ldots$, gbest $\left._{D}\right)$, which are given by

$$
\begin{gathered}
v_{i d}^{\tau+1}=w v_{i d}^{\tau}+c_{1} r_{1 d}\left(\text { pbest }_{i d}^{\tau}-x_{i d}^{\tau}\right)+c_{2} r_{2 d}\left(\text { gbest }_{d}^{\tau}-x_{i d}^{\tau}\right), \\
x_{i d}^{\tau+1}=x_{i d}^{\tau}+v_{i d}^{\tau+1},
\end{gathered}
$$

where $c_{1}$ and $c_{2}$ are acceleration constants and $r_{1 d}$ and $r_{2 d}$ are uniformly distributed random numbers in $[0,1] . w$ is the inertia weight factor.
Since PSO cannot be directly applied to multiobjective optimization, there are two major issues to be considered when extending PSO to multiobjective optimization. The first one is how to select the global and local best particles (leaders) to guide the search of a particle. The second one is how to maintain good points found so far. The algorithm based on the repository of particles [23] and mutation operator in multiobjective particles swarm optimization (MOPSO) is adopted in this paper. The steps of the MOPSO are briefly given in the following.

Step 1. Initialize the population: given the population scale $P$, randomly generate the position of each particle $P[i]$ and initialize the velocity of each particle $v[i]$ to 0 . 
Step 2. Calculate the target vector corresponding to each particle.

Step 3. Store the positions of the particles which represent nondominated vectors in the repository Rep.

Step 4. Generate hypercubes of the search space and locate the particles using these hypercubes as a coordinate system.

Step 5. Initialize the memory of each particle, which serves as a guide to travel through the search space.

Step 6. Compare current loop iteration to the maximum iterations to decide whether to continue to iterate again.

Step 6.1. Compute the speed of each particle as follows:

$$
v_{i d}^{\tau+1}=w v_{i d}^{\tau}+c_{1} r_{1 d}\left(p \text { best }_{i d}^{\tau}-x_{i d}^{\tau}\right)+c_{2} r_{2 d}\left(\operatorname{Rep}[h]_{d}^{\tau}-x_{i d}^{\tau}\right) .
$$

Different from (12), its own best position in (14) is judged by the dominance principle and the global best is replaced by Rep taken from the repository.

Step 6.2. Compute the new positions of the particles and maintain the particles within the search space.

Step 6.3. Update the contents of Rep and the geographical representation of the particles within the hypercubes.

Step 6.4. Update particles' own best position pbest $_{i}$.

Step 7. Repeat the above Steps 2 to 6 until a stopping criterion is stratified. In this paper, the MOPSO ends if the algorithm reaches the maximum amount of iterations or the maximum execution time.

\section{Numerical Results}

4.1. Array Element Antenna Design. As microstrip patch antennas have the advantages of light weight, low profile, and low cost, they are extensively utilized as the array element. With a center frequency of $3.2 \mathrm{GHz}$, a compact dual-fed stacked microstrip antenna is designed as the element of the conformal conical array. Its geometry is given in Figure 2, which is fabricated on two layers with relative permittivity of 2.65. This antenna utilizes a one-order quasi-Minkowski fractal patch on the top substrate to reduce the patch size, which also reduces the coupling between adjacent elements. The antenna is fed by standard SMA coaxial connectors from the bottom. Figure 3 shows the photograph of the fabricated antenna.

Figure 4 displays the measured and simulated impedance bandwidth for the antenna. The measured impedance bandwidth (VSWR $\leq 2)$ by Agilent N5230A network analyzer is from 3.0 to $3.61 \mathrm{GHz}(18.46 \%)$. There is a good agreement between simulated and measured results. Therefore, the designed antenna can fully satisfy the demanded requirements.

For conformal arrays, to determine the total radiation pattern produced at a far-field point, it is more convenient

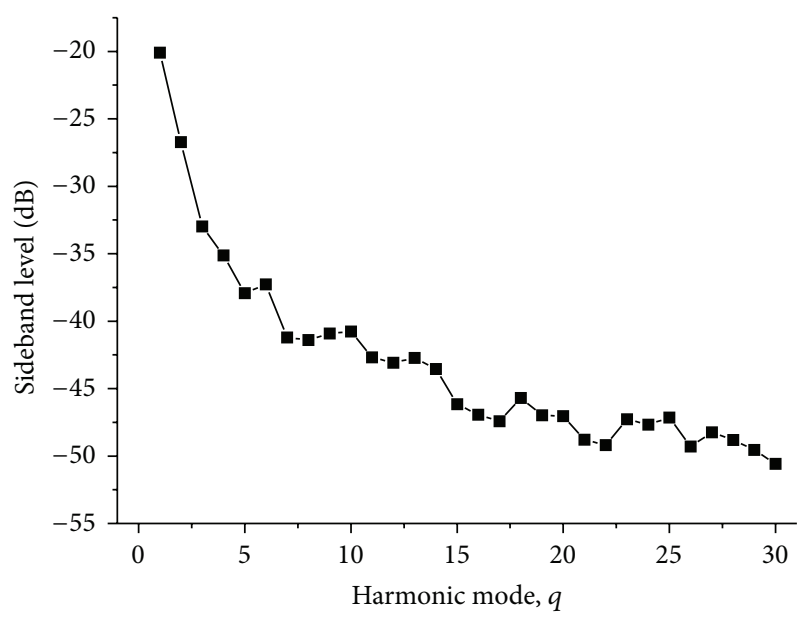

Figure 10: The maximum sideband levels of the first 30 positive harmonics for scan direction of $\left(74^{\circ}, 0^{\circ}\right)$ for solution A in Figure 7.

to consider the field of individual elements in their own coordinate systems firstly and then transform the field back to the global coordinate system [24].

4.2. Pattern Synthesis. Since polarization plays a very important role in conformal phased arrays, the polarization radiation pattern is investigated in this paper. To reduce the crosspolarization of the conical array, the technique based on combining the weighted individual feeding ports of the patch antennas before beamforming is employed in this paper [25].

As shown in Figure 5, each antenna is fed using dual feeds. For the $n$th radiator, the $\theta$-feed point will excite two radiated fields, that is, a copolarized field $E_{n \theta}^{\theta}$, in the $\hat{\theta}$-direction and a cross-polarized component $E_{n \varphi}^{\theta}$. Similarly, the $\varphi$-feed point will result in a copolarized radiated field $E_{n \varphi}^{\varphi}$ in the $\widehat{\varphi}$ direction and a cross-polarized component $E_{n \theta}^{\varphi}$. Besides, the polarization angle $\left(\gamma_{d}\right)$ for linear polarization is defined as the angle between the $\widehat{\theta}$-unit vector and the unit vector in the direction of the desired polarization at the field point in the direction of the main beam, as shown in Figure 6.

For the $n$th element, the unit vector of the desired polarization is a linear combination of the $\theta$ - and $\varphi$-components of the radiated field in the direction of the main beam as follows:

$$
\begin{aligned}
& E_{n}\left(\theta_{d}, \varphi_{d}\right) \sin \gamma_{d}=\alpha_{n} E_{n \varphi}^{\theta}\left(\theta_{d}, \varphi_{d}\right)+\beta_{n} E_{n \varphi}^{\varphi}\left(\theta_{d}, \varphi_{d}\right), \\
& E_{n}\left(\theta_{d}, \varphi_{d}\right) \cos \gamma_{d}=\alpha_{n} E_{n \theta}^{\theta}\left(\theta_{d}, \varphi_{d}\right)+\beta_{n} E_{n \theta}^{\varphi}\left(\theta_{d}, \varphi_{d}\right),
\end{aligned}
$$

where $E_{n}\left(\theta_{d}, \varphi_{d}\right)$ is the desired polarization from the $n$th radiator in the direction of the main beam. $E_{n \varphi}^{\theta}\left(\theta_{d}, \varphi_{d}\right)$ and $E_{n \varphi}^{\varphi}\left(\theta_{d}, \varphi_{d}\right)$ are the $\varphi$-components of the radiated field from $\theta$ - and $\varphi$-feed point of the $n$th radiator, respectively. Whereas, $E_{n \theta}^{\theta}\left(\theta_{d}, \varphi_{d}\right)$ and $E_{n \theta}^{\varphi}\left(\theta_{d}, \varphi_{d}\right)$ are the $\theta$-components from $\theta$ - and $\varphi$-feed point of the $n$th radiator, respectively. $\alpha_{n}$ and $\beta_{n}$ are, respectively, the weights for the $\theta$ - and $\varphi$-feed point of the $n$th 


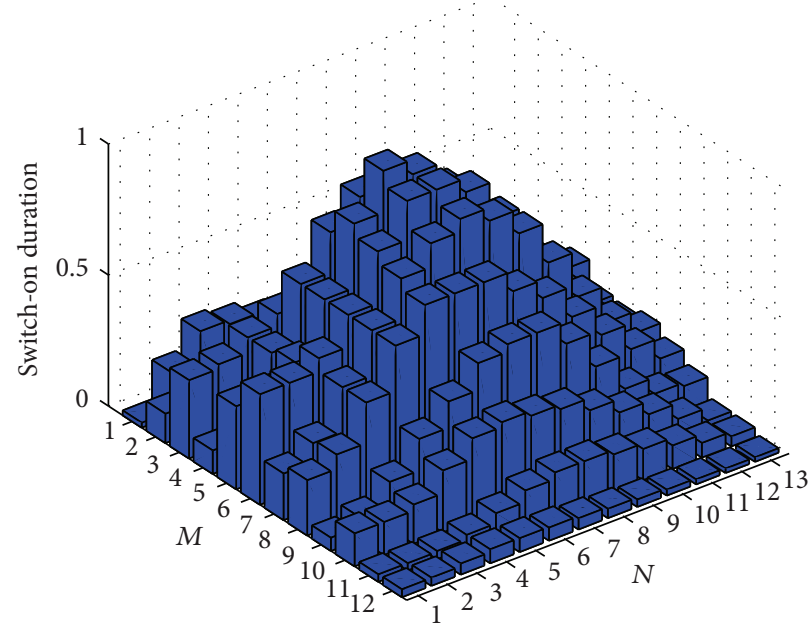

(a) Theta feed point

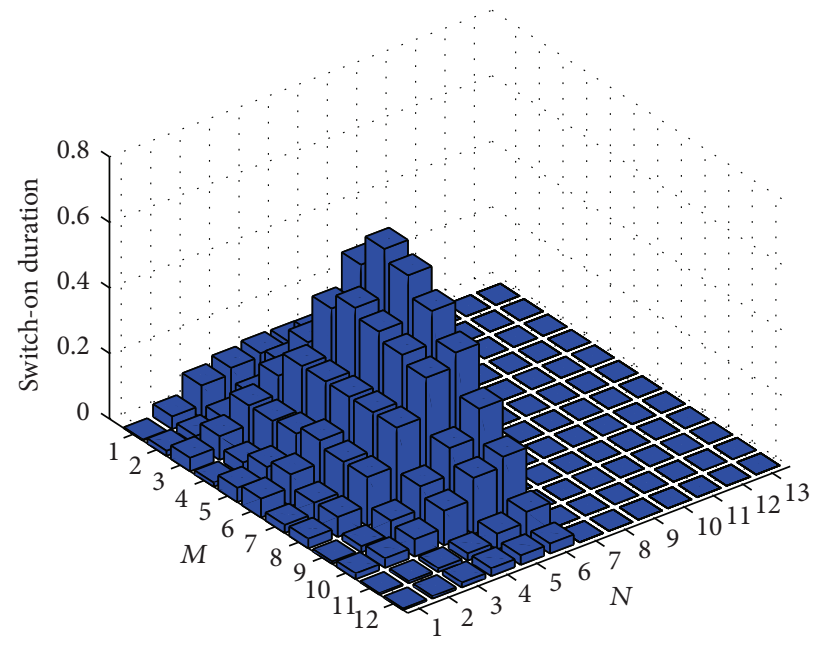

(b) Phi feed point

Figure 11: Optimized switch-on durations in the theta feed point and phi feed point for scan direction of $\left(74^{\circ}, 0^{\circ}\right)$ for solution A in Figure 7 .

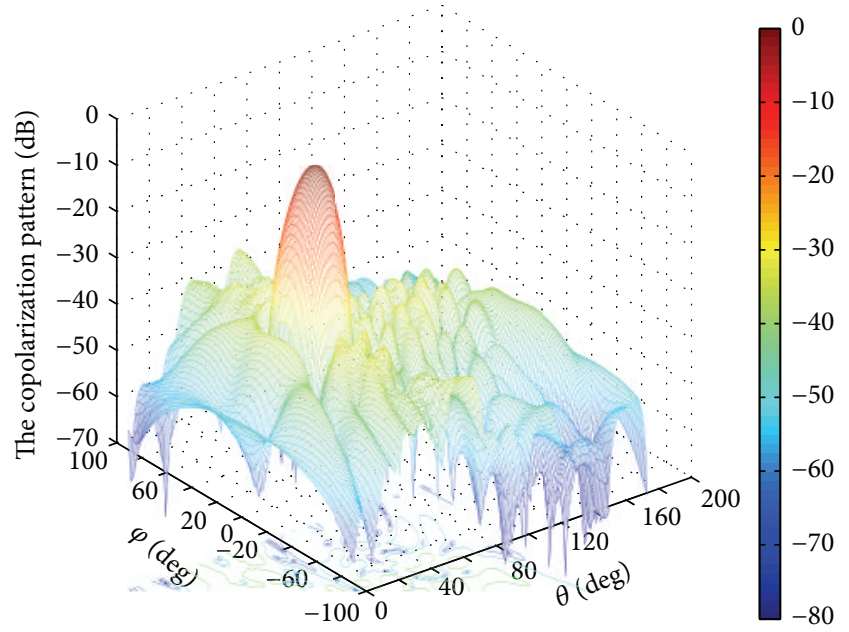

(a) Copolarization pattern

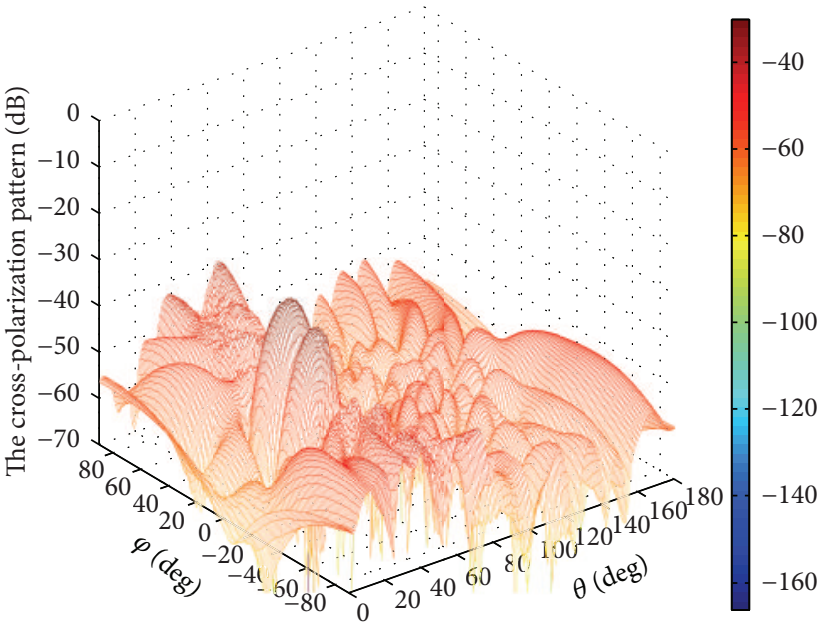

(b) Cross-polarization pattern

Figure 12: 3D radiation pattern of the conical conformal array for scan direction of $\left(45^{\circ}, 0^{\circ}\right)$ for solution A in Figure 7.

radiator to achieve the required polarization in the direction of the main beam. From (15) we can derive that

$$
\tan \gamma_{d}=\frac{\alpha_{n} E_{n \varphi}^{\theta}\left(\theta_{d}, \varphi_{d}\right)+\beta_{n} E_{n \varphi}^{\varphi}\left(\theta_{d}, \varphi_{d}\right)}{\alpha_{n} E_{n \theta}^{\theta}\left(\theta_{d}, \varphi_{d}\right)+\beta_{n} E_{n \theta}^{\varphi}\left(\theta_{d}, \varphi_{d}\right)} .
$$

From (16), the ratio of the excitation amplitude between the two feed ports can be written as

$$
\frac{\beta_{n}}{\alpha_{n}}=\frac{\tan \gamma_{d} E_{n \theta}^{\theta}\left(\theta_{d}, \varphi_{d}\right)-E_{n \varphi}^{\theta}\left(\theta_{d}, \varphi_{d}\right)}{E_{n \varphi}^{\varphi}\left(\theta_{d}, \varphi_{d}\right)-\tan \gamma_{d} E_{n \theta}^{\varphi}\left(\theta_{d}, \varphi_{d}\right)} .
$$

Thus, before the synthesis of the radiation patterns, the excitation relation between the two feed ports can be determined, which can be used to effectively reduce half of the optimization variables.

The arrays considered here are fed with a uniform static excitation $\left(\left|I_{n}\right|=1\right)$, a target of which is always desired in practice. For conformal arrays, it is common to select the excitation phase to focus the beam in the desired direction. With the phase excitation calculated by (2), the specified scan angle can be guaranteed. Only the pulse durations are considered in the numerical simulation later. In this paper, a $12 \times 13$ conical conformal phased array is investigated; that is, the array consists of 156 elements. Now considering that the optimization for the pulse durations of each element on the conical phased array may be a prohibitive task in practical engineering, then, a previously proposed modified Bernstein 


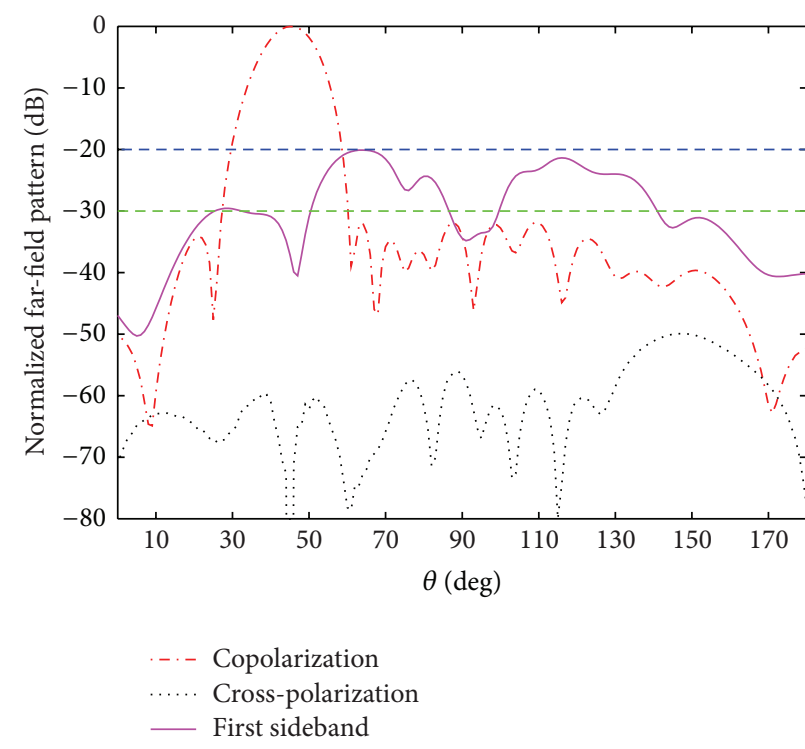

(a) $\varphi=0^{\circ}$ plane

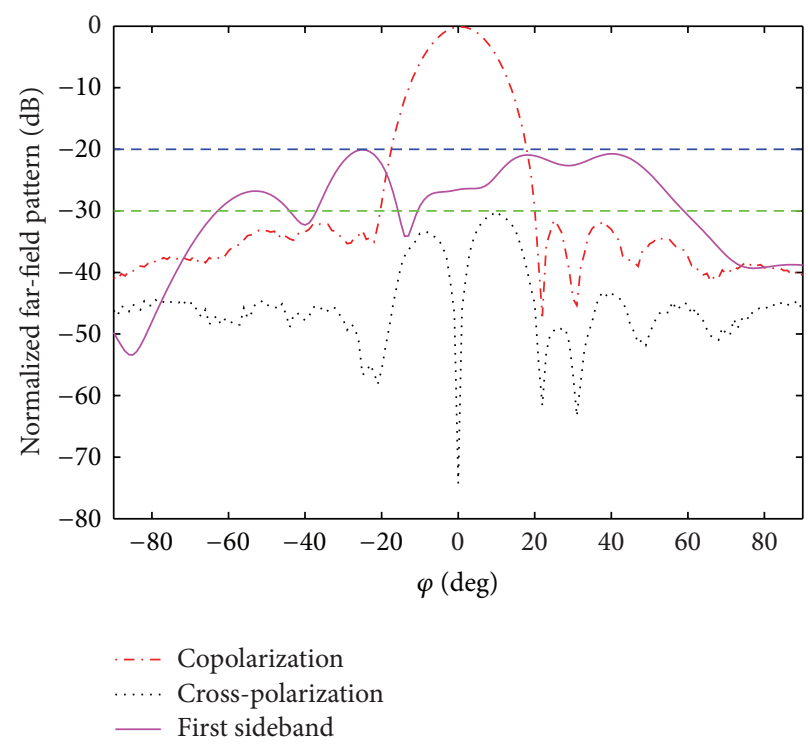

(b) $\theta=45^{\circ}$ plane

FIGURE 13: Sectional views in the elevation and azimuth planes for scan direction of $\left(45^{\circ}, 0^{\circ}\right)$ for solution A in Figure 7.

polynomial for arc arrays [5] is extended to 3D case to be used. The modified Bernstein polynomial is defined as

$$
F(U)=\left\{\begin{aligned}
& B_{1}+\frac{1-B_{1}}{A^{N_{0} A}(1-A)^{N_{0}(1-A)}} U^{N_{0} A}(1-U)^{N_{0}(1-A)}, \\
& 0 \leq U \leq A \\
& B_{2}+\frac{1-B_{2}}{A^{N_{1} A}(1-A)^{N_{1}(1-A)}} U^{N_{1} A}(1-U)^{N_{1}(1-A)}, \\
& A \leq U \leq 1,
\end{aligned}\right.
$$

where $B_{1}, B_{2}, M_{1}, M_{2}$, and $A$ are parameters in the polynomial. By using the modified Bernstein polynomial, in our pattern synthesis example only five variables need to be optimized of each concentric ring. The differences among the maximum amplitude of each row are also obtained by a modified Bernstein polynomial. That is to say, for a spherical conformal array constituting of $M$ concentric circular arrays, the total number of variables to be optimized can be reduced to $5 \times(M+1)$, which can significantly alleviate the burden of the algorithm in the optimization process. For the conical conformal array consisting of 12 rows, the total number of variables to be optimized is reduced from $12 \times 13$ to $5 \times(12+$ 1); that is, the optimization variables can be reduced by $58.3 \%$.

In this paper, the third definition of Ludwig is used to define the copolarization $E_{\mathrm{co}}$ and the cross-polarization $E_{\text {cross }}$ [26] as follows:

$$
\begin{gathered}
E_{\text {co }}=E_{\theta} \cos \varphi-E_{\varphi} \sin \varphi, \\
E_{\text {cross }}=E_{\theta} \sin \varphi+E_{\varphi} \cos \varphi .
\end{gathered}
$$

To illustrate the effectiveness of the proposed mechanism, the three-dimensional $(3 \mathrm{D})$ radiation patterns of the array are synthesized in three scan angles: $\left(74^{\circ}, 0^{\circ}\right),\left(45^{\circ}, 0^{\circ}\right)$, and $\left(105^{\circ}, 0^{\circ}\right)$. For this time-modulated conformal array, we focus on achieving low sidelobe level and low sideband level simultaneously as follows:

$$
\min \operatorname{FF}(\vec{x})=\{\operatorname{SLL}(\vec{x}), \operatorname{SBL}(\vec{x})\} .
$$

Behavior of the MOPSO on the considered synthesis problem has been investigated. Simulation results show that to ensure the convergence of the optimization process, parameters in (14) should satisfy $0.5\left(c_{1}+c_{2}\right)<1.5(1+w)$ condition and in such case the population mean converges to the desired solution set. Otherwise, the algorithm may fail to converge to the desired solution set. Thus, in the numerical experiments, the inertia weight factor $w$ is chosen to be 0.4 , the acceleration constants $c_{1}=c_{2}=1$. The population size is set to 100 , the repository size $r p=100$, the mutation rate $m u=0.5$, and the maximum number of generations is set to 2000 . The proposed approach runs on an I7-2620 $2.7 \mathrm{GHz}$ CPU with 4 GB memory. The simulation times for different scan angles are roughly the same, which is about 22.32 minutes. The distribution of the Pareto front (PF) achieved by the MOPSO for scan direction of $\left(74^{\circ}, 0^{\circ}\right)$ is shown in Figure 7. The coordinate values of the axes represent the maximum SLL (MSLL) at $f_{0}$ and the maximum SBL (MSBL). Each point of the Pareto front represents a feasible design case, which can provide great freedom to the designers, as compared with the single-objective optimization techniques. To further validate the effectiveness of the proposed method, the normalized radiation patterns for one of the solutions, solution A (in Figure 7), are plotted, as presented in Figure 8. The copolarization and cross-polarization patterns and the first sideband cuts through the main beam at $\varphi=0^{\circ}$ and $\theta=74^{\circ}$ are illustrated in Figure 9. From those simulation results, it can be seen that the main beam points in the desired direction with sidelobe levels are below $-30 \mathrm{~dB}$ and 


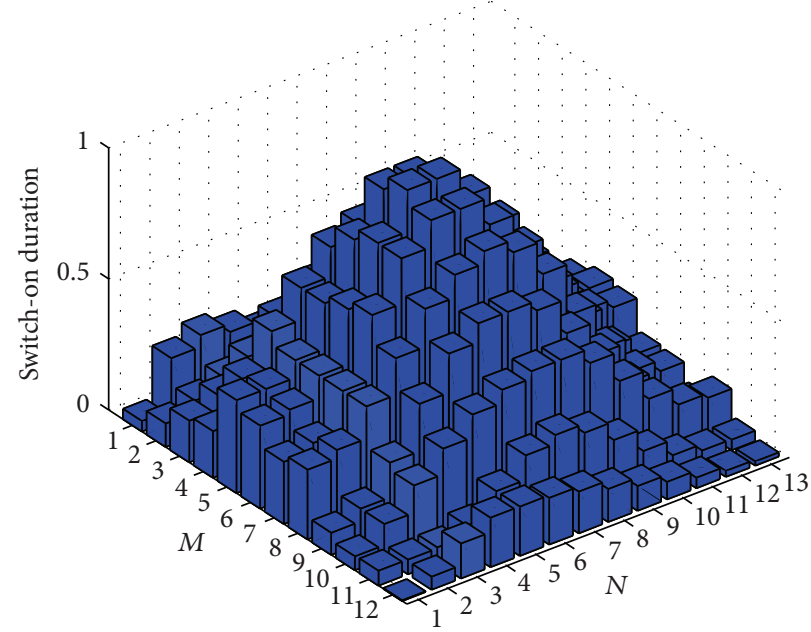

(a) Theta feed point

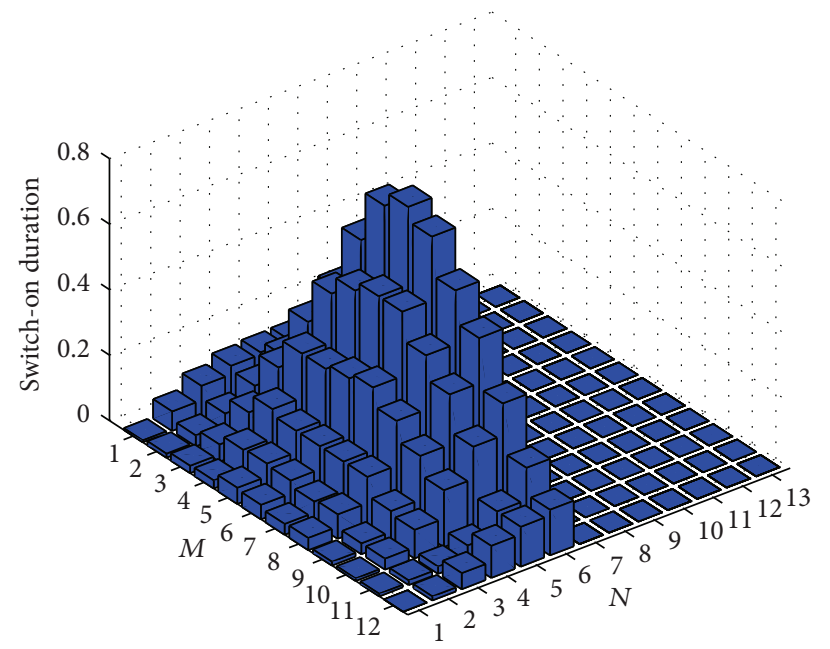

(b) Phi feed point

FIGURE 14: Optimized switch-on durations in the theta feed point and phi feed point for scan direction of $\left(45^{\circ}, 0^{\circ}\right)$ for solution A in Figure 7 .

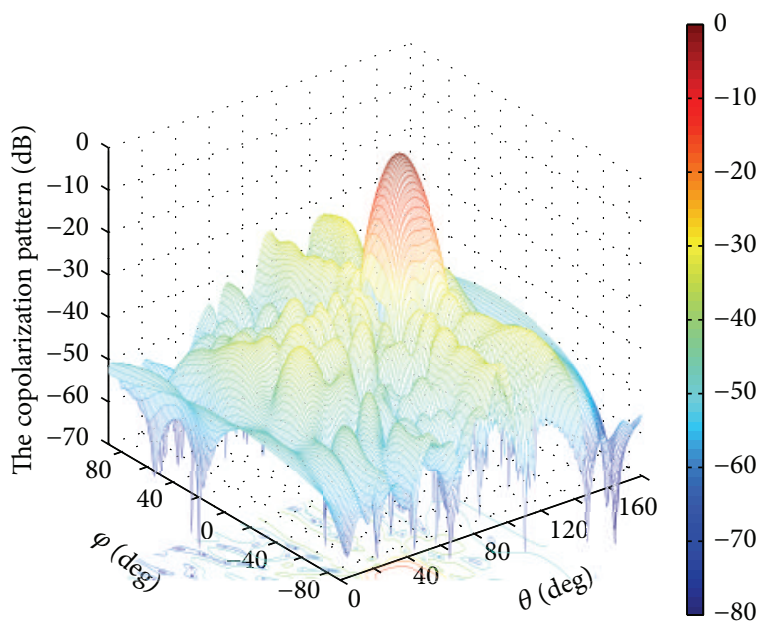

(a) Copolarization pattern

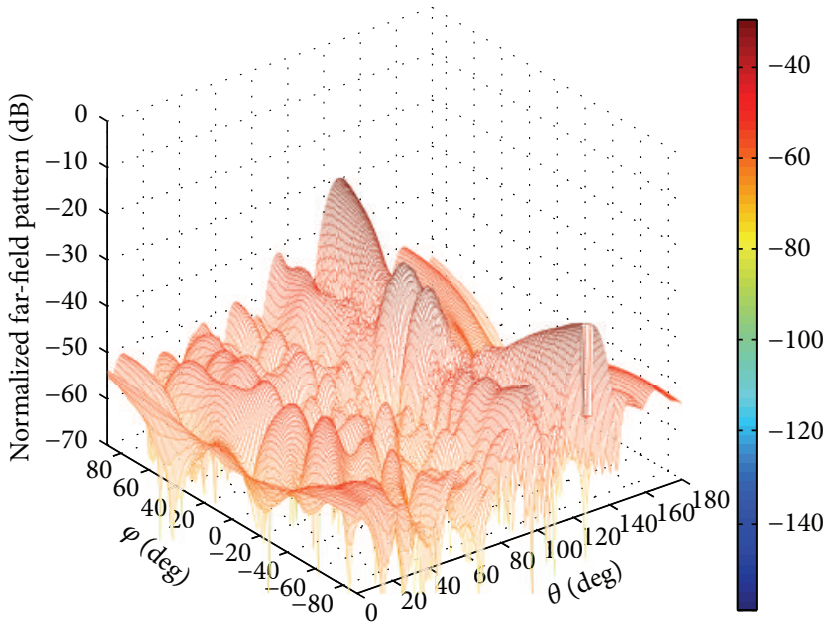

(b) Cross-polarization pattern

FIGURE 15: 3D radiation pattern of the conical conformal array for scan direction of $\left(105^{\circ}, 0^{\circ}\right)$ for solution A in Figure 7.

the cross-polarization pattern for the array are also below $-30 \mathrm{~dB}$. Furthermore, the MSBL for the first sidebands is $-20.08 \mathrm{~dB}$, which can also be seen from Figures 8 and 9. In order to prove the suppression of SBL, the maximum levels of first 30 harmonics in this direction are provided in Figure 10. For limited space, the figures corresponding to the other two scan angles are not provided because their results are similar to that in Figure 10. Figure 11 provides the optimized pulse duration sequences for the dual-fed stacked microstrip elements in theta feed point and phi feed point.

Due to the similar distributions of the Pareto front (PF) for scan directions of $\left(45^{\circ}, 0^{\circ}\right)$ and $\left(105^{\circ}, 0^{\circ}\right)$ as in Figure 7 , they are not presented. Also, one of the solutions for these two scan directions chosen from the Pareto front is plotted. The $3 \mathrm{D}$ radiation patterns for these two scan angles are presented in Figures 12 and 15, respectively. The corresponding copolarization and cross-polarization patterns and first sideband cuts through the main beams are illustrated in Figures 13 and 16, respectively. From the figures, we can also discover that sidelobe levels are all below $-30 \mathrm{~dB}$ and the cross-polarization patterns also below $-30 \mathrm{~dB}$. The required pulse duration sequences for the dual-fed stacked microstrip elements in theta feed point and phi feed point for these two scan angles are shown in Figures 14 and 17, respectively.

\section{Conclusion}

This paper has presented time-modulated conformal phased array synthesis optimization by using the multiobjective particle swarm optimization. Conflicting specifications such 

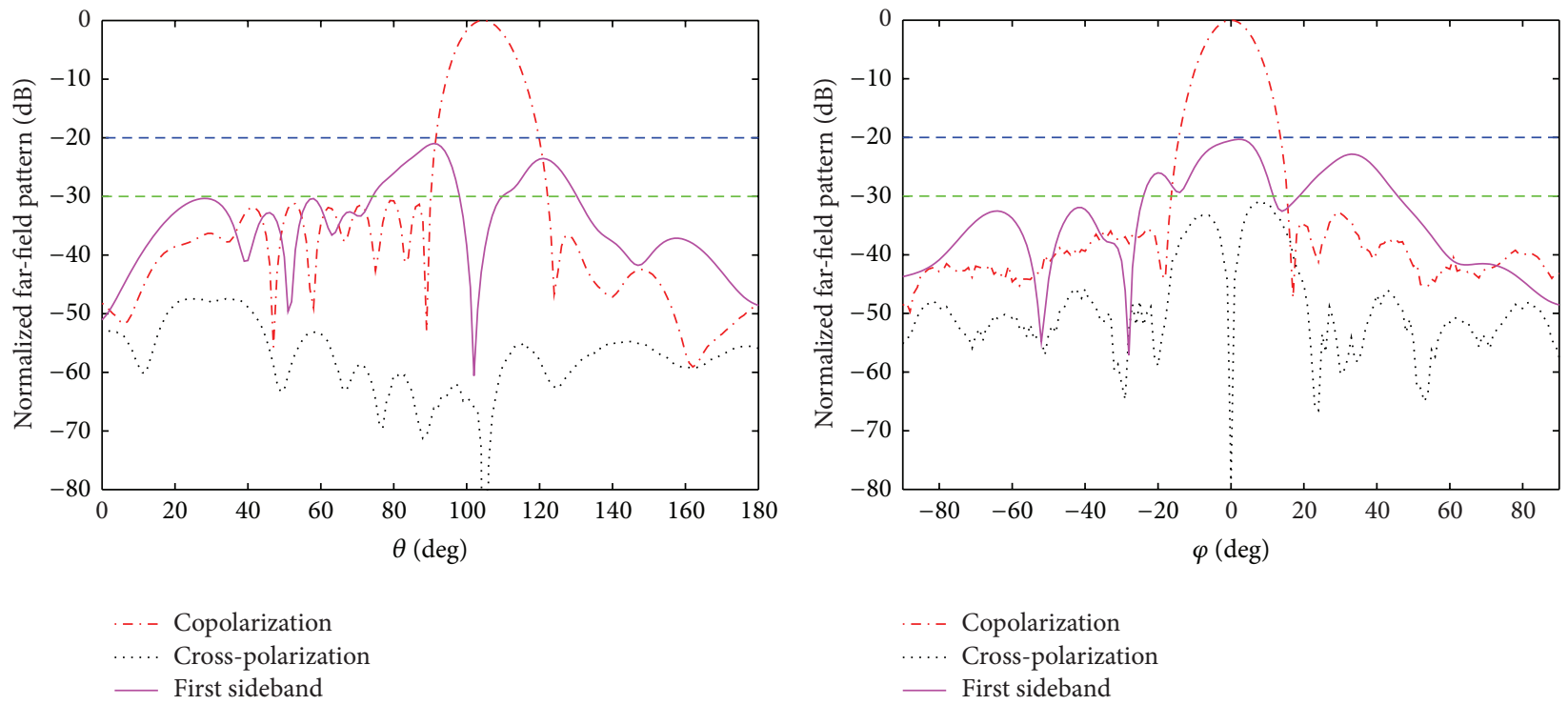

$\begin{array}{ll}\text {...... Copolarization } & \text { Cross-polarization }\end{array}$

_ First sideband

_ First sideband

(a) $\varphi=0^{\circ}$ plane

(b) $\theta=105^{\circ}$ plane

FIgURE 16: Sectional views in the elevation and azimuth planes for scan direction of $\left(105^{\circ}, 0^{\circ}\right)$ for solution A in Figure 7.

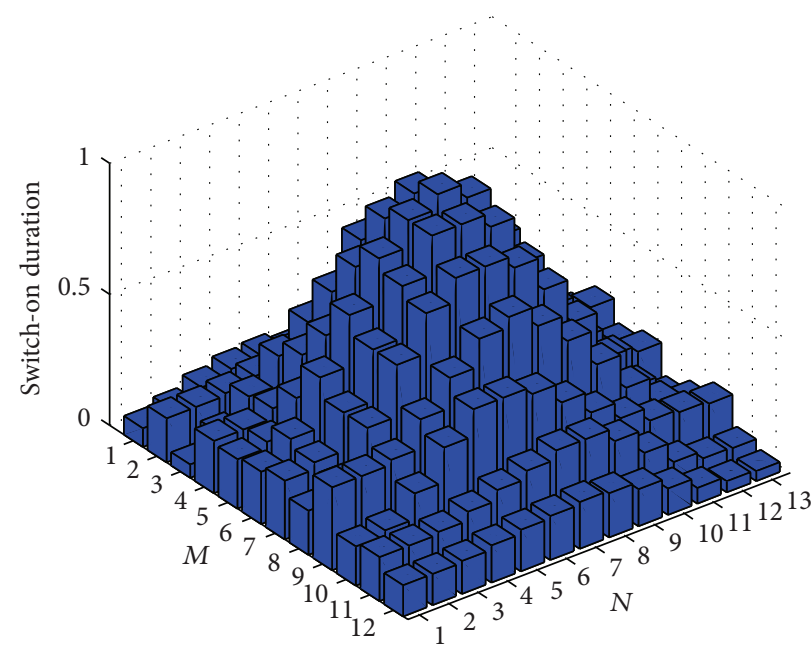

(a) Theta feed point

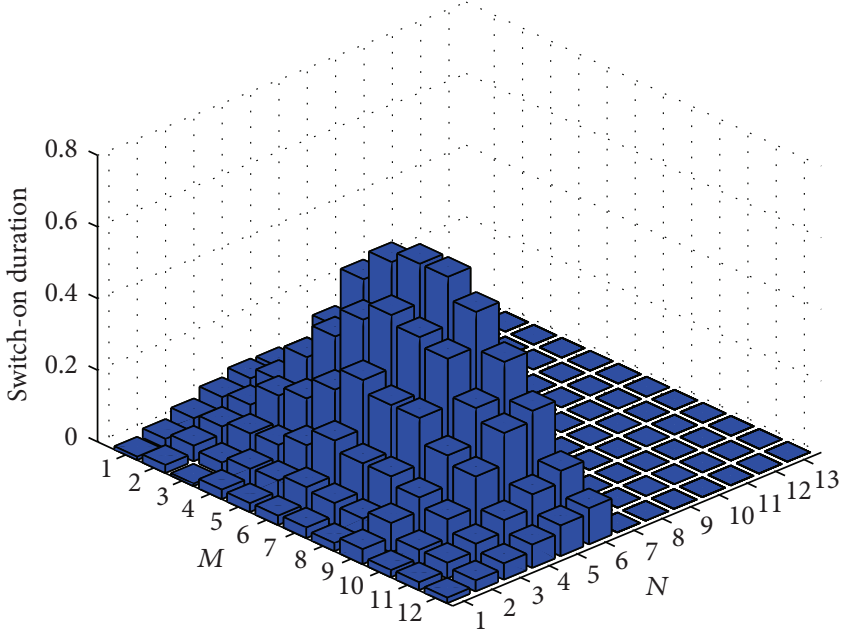

(b) Phi feed point

Figure 17: Optimized switch-on durations in the theta feed point and phi feed point for scan direction of $\left(105^{\circ}, 0^{\circ}\right)$ for solution A in Figure 7 .

as the peak SLL and SBL have been optimized simultaneously. Additionally, the modified Bernstein polynomial is extended to $3 \mathrm{D}$ case in synthesizing conformal arrays in order to greatly reduce the optimization variables. Dual fed patch antennas are designed as the radiating elements to achieve low crosspolarization level during the beam scanning. The proposed algorithm has been successfully applied to design a $12 \times 13$ element conical conformal microstrip phased array, which can provide great freedom to the designers as compared with the single-objective optimization techniques. The numerical results reveal the promising characteristics of the mechanism in synthesizing time-modulated conformal arrays.

\section{Conflict of Interests}

The authors declare that there is no conflict of interests regarding to the publication of this paper.

\section{Acknowledgments}

The authors would like to thank the financial support from the Natural Science Foundation of China (no. 61101069 and no. 61201135) and the Fundamental Research Funds for the Central Universities (no. K5051302022 and no. K7214569601). 


\section{References}

[1] L. Josefsson and P. Persson, Conformal Array Antenna Theory and Design, John Wiley \& Sons, New York, NY, USA, 2006.

[2] K. Wincza, S. Gruszczynski, and K. Sachse, "Conformal fourbeam antenna arrays with reduced sidelobes," Electronics Letters, vol. 44, no. 3, pp. 174-175, 2008.

[3] Y. Y. Bai, S. Xiao, C. Liu, and B. Z. Wang, "A hybrid IWO/PSO algorithm for pattern synthesis of conformal phased arrays," IEEE Transactions on Antennas and Propagation, vol. 61, no. 4, pp. 2328-2332, 2013.

[4] O. M. Bucci, A. Capozzoli, and G. D’Elia, “Power pattern synthesis of reconfigurable conformal arrays with near-field constraints," IEEE Transactions on Antennas and Propagation, vol. 52, no. 1, pp. 132-141, 2004.

[5] D. W. Boeringer and D. H. Werner, "Efficiency-constrained particle swarm optimization of a modified Bernstein polynomial for conformal array excitation amplitude synthesis," IEEE Transactions on Antennas and Propagation, vol. 53, no. 8, pp. 2662-2673, 2005.

[6] L. I. Vaskelainen, "Constrained least-squares optimization in conformal array antenna synthesis," IEEE Transactions on Antennas and Propagation, vol. 55, no. 3, pp. 859-867, 2007.

[7] B. Fuchs, "Shaped beam synthesis of arbitrary arrays via linear programming," IEEE Antennas and Wireless Propagation Letters, vol. 8, pp. 481-484, 2010.

[8] K. M. Tsui and S. C. Chan, "Pattern synthesis of narrowband conformal arrays using iterative second-order cone programming," IEEE Transactions on Antennas and Propagation, vol. 58, no. 6, pp. 1959-1970, 2010.

[9] H. E. Shanks and R. W. Bickmore, "Four-dimensional electromagnetic radiators," Canadian Journal of Physics, vol. 37, no. 3, pp. 263-275, 1959.

[10] L. Poli, P. Rocca, L. Manica, and A. Massa, "Handling sideband radiations in time-modulated arrays through particle swarm optimization," IEEE Transactions on Antennas and Propagation, vol. 58, no. 4, pp. 1408-1411, 2010.

[11] J. Fondevila, J. C. Brégains, F. Ares, and E. Moreno, "Optimizing uniformly excited linear arrays through time modulation," IEEE Antennas and Wireless Propagation Letters, vol. 3, no. 1, pp. 298301, 2004

[12] S. W. Yang, Y. B. Gan, and A. Y. Qing, "Sideband suppression in time-modulated linear arrays by the differential evolution algorithm," IEEE Antennas and Wireless Propagation Letters, vol. 1, pp. 173-175, 2002.

[13] S. K. Mandal, R. Ghatak, and G. K. Mahanti, "Minimization of side lobe level and side band radiation of a uniformly excited time modulated linear antenna array by using artificial bee colony algorithm," in Proceedings of the IEEE Symposium on Industrial Electronics and Applications (ISIEA '11), pp. 247-250, September 2011.

[14] Q. Zhu, S. Yang, R. Yao, and Z. Nie, "Gain improvement in timemodulated linear arrays using SPDT switches," IEEE Antennas and Wireless Propagation Letters, vol. 11, pp. 994-997, 2012.

[15] Y. Wang and A. Tennant, "Time-modulated reflector array," Electronics Letters, vol. 48, no. 16, pp. 972-974, 2012.

[16] E. T. Bekele, L. Poli, P. Rocca, M. DUrso, and A. Massa, "Pulse-shaping strategy for time modulated arrays-analysis and design," IEEE Transactions on Antennas and Propagation, vol. 61, no. 7, pp. 3525-3537, 2013.

[17] E. Aksoy and E. Afacan, "Calculation of sideband power radiation in time-modulated arrays with asymmetrically positioned pulses,' IEEE Antennas and Wireless Propagation Letters, vol. 11, pp. 133-136, 2012.

[18] A. Tennant, "Experimental two-element time-modulated direction finding array," IEEE Transactions on Antennas and Propagation, vol. 58, no. 3, pp. 986-988, 2010.

[19] G. Li, S. Yang, and Z. Nie, "Direction of arrival estimation in time modulated linear arrays with unidirectional phase center motion," IEEE Transactions on Antennas and Propagation, vol. 58, no. 4, pp. 1105-1111, 2010.

[20] E. Aksoy and E. Afacan, "An inequality for the calculation of relative maximum sideband level in time-modulated linear and planar arrays," IEEE Transactions on Antennas and Propagation, vol. PP, no. 99, 2014.

[21] K. Deb, Multiobjective Evolutionary Algorithm, John Wiley \& Sons, Chichester, UK, 2001.

[22] R. Eberhart and J. Kennedy, "New optimizer using particle swarm theory," in Proceedings of the 6th International Symposium on Micro Machine and Human Science, pp. 39-43, October 1995.

[23] C. A. C. Coello, G. T. Pulido, and M. S. Lechuga, "Handling multiple objectives with particle swarm optimization," IEEE Transactions on Evolutionary Computation, vol. 8, no. 3, pp. 256-279, 2004.

[24] H. A. Burger, "Use of euler-rotation angles for generating antenna pattern," IEEE Antennas and Propagationg Magazine, vol. 37, no. 2, pp. 56-63, 1995.

[25] C. Dohmen, J. W. Odendaal, and J. Joubert, "Synthesis of conformal arrays with optimized polarization," IEEE Transactions on Antennas and Propagation, vol. 55, no. 10, pp. 2922-2925, 2007.

[26] A. C. Ludwig, "The definition of cross polarization," IEEE Transactions on Antennas and Propagation, vol. AP-21, no. 1, pp. 116-119, 1973. 

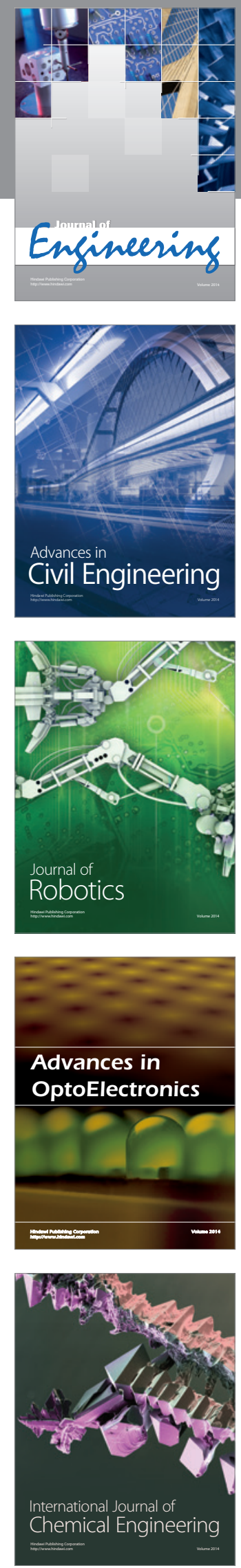

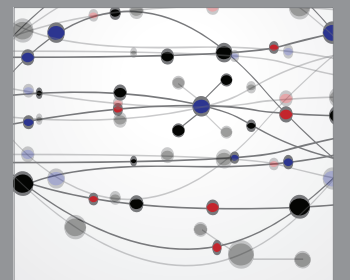

The Scientific World Journal
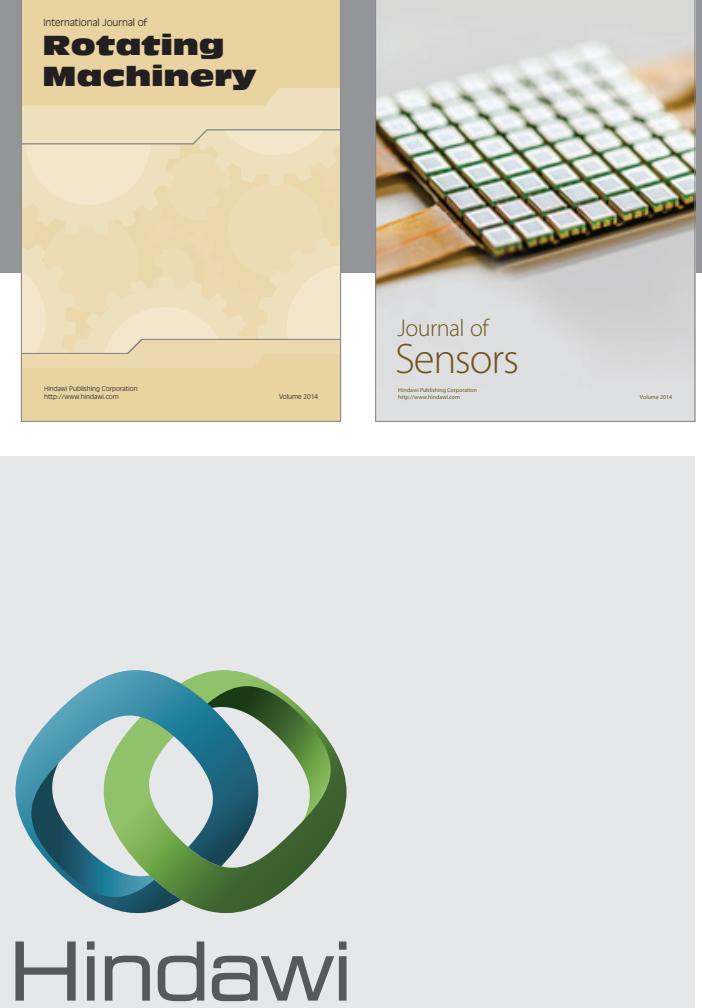

Submit your manuscripts at http://www.hindawi.com
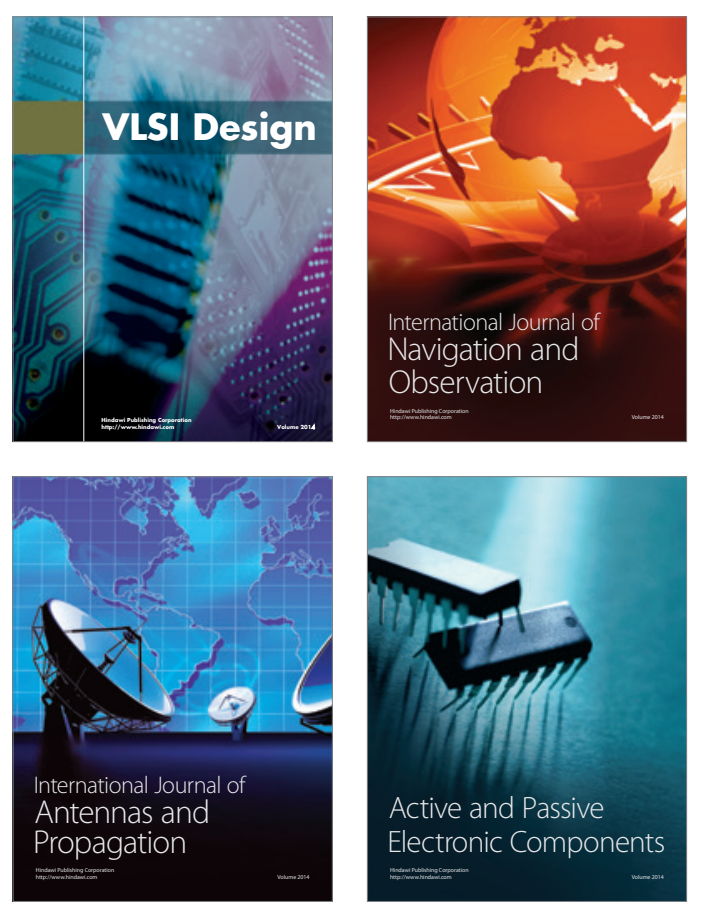
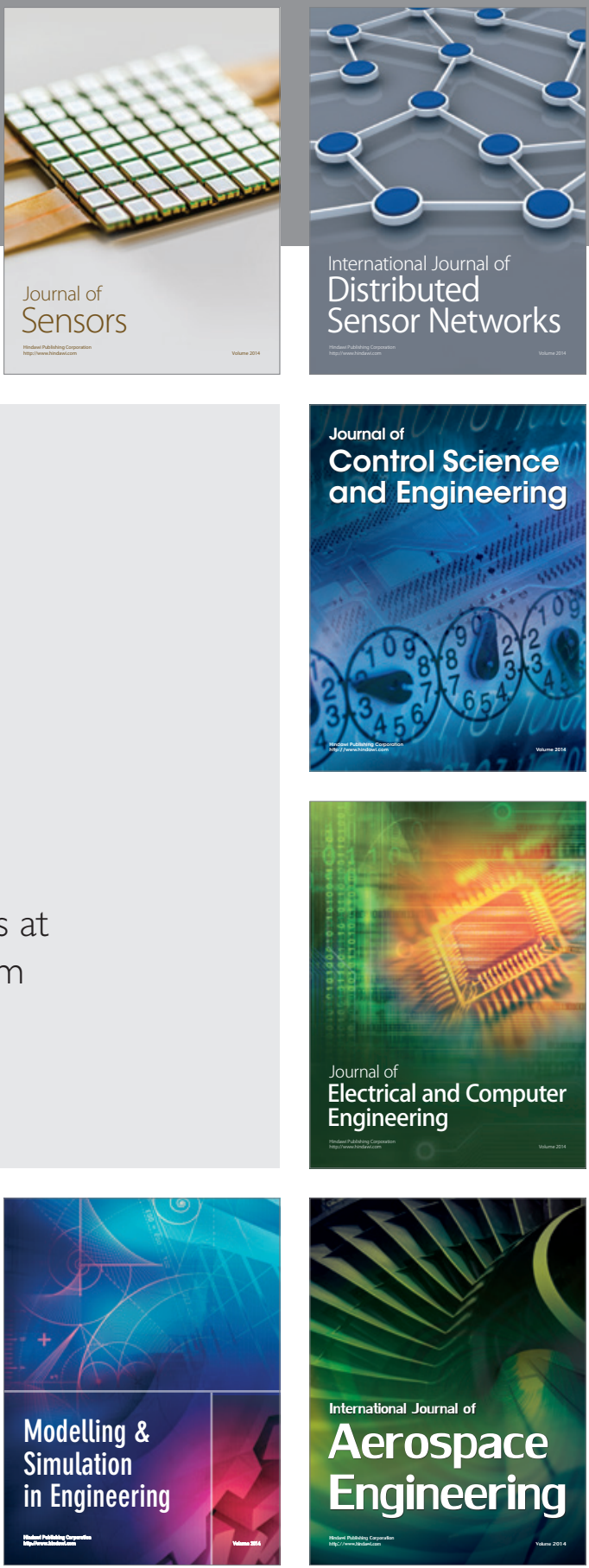

Journal of

Control Science

and Engineering
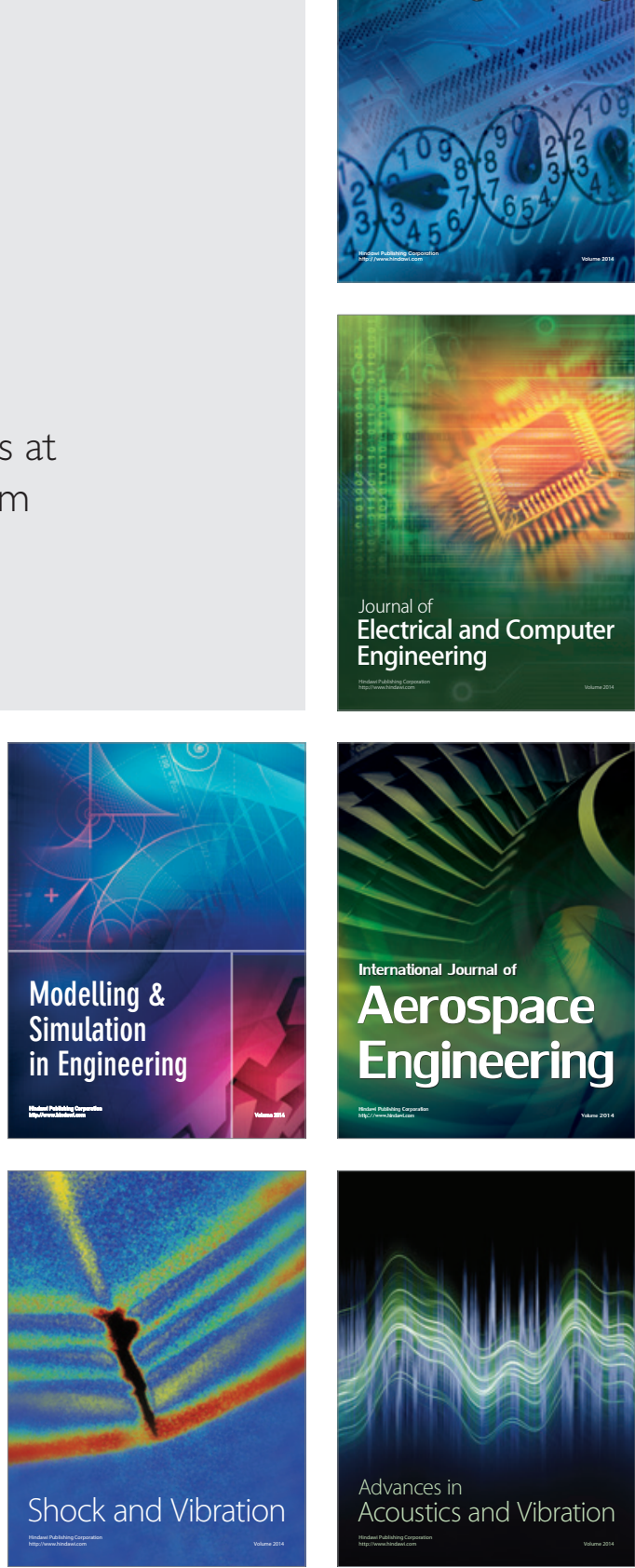